\title{
Kinetic Energy Penetrator (KEP) impact on confined concrete
}

\author{
Hakim Abdulhamid ${ }^{1}$, Kevin Delabre ${ }^{1}$, Fabien Plassard $^{1}$, Pierre Héreil ${ }^{1}$, Jérôme Mespoulet $^{1}$ \\ and Paul Deconinck ${ }^{1}$
}

${ }^{1}$ Shock Physics Department, THIOT INGENIERIE, 830 Route Nationale, 46130 Puybrun, France

\begin{abstract}
Understanding concrete response facing warheads threats is important for both the design of strategic infrastructure protection and the prediction of warhead performances. This ongoing study aims at building a robust approach for the characterisation of concrete behaviour under ballistic impact of Kinetic Energy Penetrator (KEP). A set of tests has been developed and performed to fit the main parameters of the Holmquist Johnson Cook Concrete material model. Highly instrumented tests are conducted to improve the model prediction capability and to identify its limits. After a brief description of the test configuration, the paper focuses on the analysis of an impact test and presents preliminary simulation results.
\end{abstract}

\section{Introduction}

Design of strategic infrastructures requires the evaluation of its performance against warhead threat. Similarly, the question may also be raised for existing buildings as threats evolve. Predicting the response of concrete under such ballistic penetration and/or blast events requires a model with a good representation of some non-linear phenomena like compaction, cracks and spalling. Due to its rock-like behaviour, concrete material shows unconventional mechanical behaviour since its response is highly depended on pressure (higher pressure leads to higher strength). Numerical simulations of such events are quite challenging. Meshing choice is for instance important; for example, using finite elements may affect the compaction behaviour in case of element erosion. Another difficulty for conducting simulation is related to the unavailability of a full set of material. Dynamic characterization data and ballistic tests results are not generally available for a specific concrete. Characteristics of two different batch of concrete may differ especially as they evolve in time and with storage conditions.

Focusing on a KEP impacting a highly confined concrete, this study aims at developing and validating an approach for the development of a concrete model able to predict such event. The model should require a reasonable amount of data for it to be usable in an industrial context. The Holmquist-Johnson-Cook Concrete model (HJC) [1] available in IMPETUS $\mathrm{AFEA}^{\mathrm{TM}}$ is used. To reduce some sources of uncertainties, both dynamic characterisation tests and ballistic impact are realised. All the specimens of the study have been manufactured from the same batch. The concrete is modelled with recent type of meshless method called $\gamma$-SPH that reveals its efficiency in fragmentation and hypervelocity impacts [2]. This paper shows some preliminary results for a ballistic non-perforating impact and its simulation. 


\section{Concrete modelling}

The HJC model has been chosen for its limited number of parameters to reduce the necessary characterisation tests. It can be summarized with two main equations: volume strain response (EOS) represented by a pressure compaction relationship and the strength vs pressure dependency of intact and damage concrete. The major phenomena during impact which are the large compaction around the projectile nose and possible spalling of the target back side can therefore be fairly represented. The concrete specimen is represented with $\gamma$-SPH elements and the remaining parts are modelled with finite elements.

\section{KE penetrator impact test and simulation}

The test consists in an impact of a $520 \mathrm{~g} \mathrm{KEP}$ on a cylindrical concrete specimen $100 \mathrm{~mm}$ diameter and $200 \mathrm{~mm}$ length. The specimen is inside a metallic jacket of $5 \mathrm{~mm}$ thickness to represent the confinement provided by concrete reinforcements. The impact velocity is 400 $\mathrm{m} / \mathrm{s}$. A high-speed camera records the event from the top side of the target.
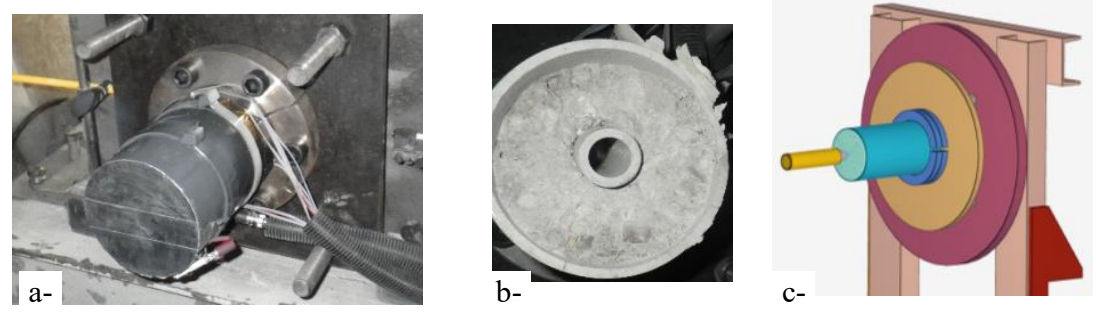

Fig. 1. a-Test configuration, b-post-mortem image of the target, c- numerical model
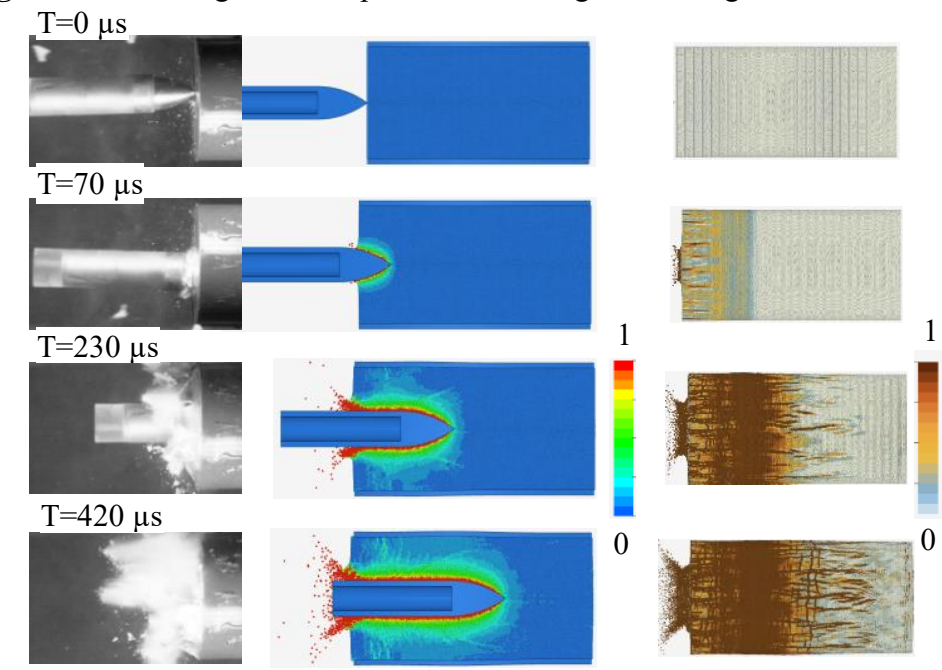

$\mathrm{T}=900 \mu \mathrm{s}$
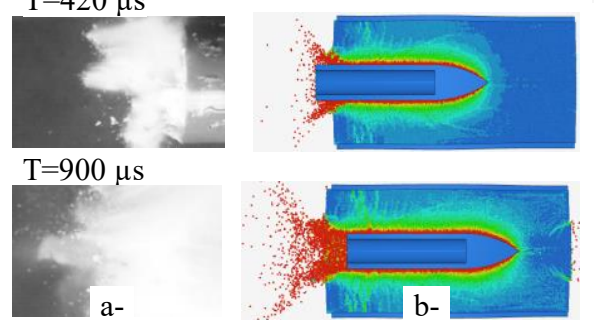

0
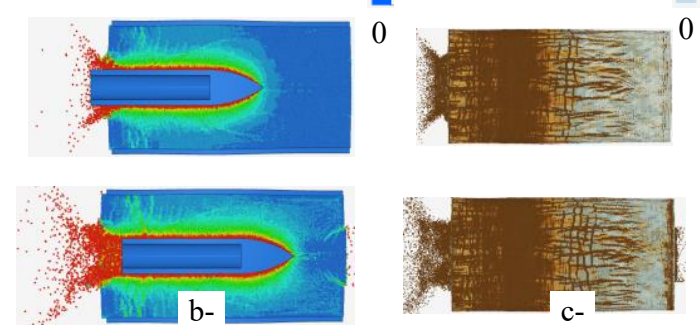

Fig. 2. Comparison of test with simulation: a-Images from high speed camera, b-Simulated concrete internal strain, c-Simulated concrete external damage

Fig. 2 compare some experimental data with the simulation. As observed, the front side of the concrete is completely fragmented and ejected from its jacket. The penetrator did not completely perforate the target, the depth of penetration is $160 \mathrm{~mm}$. The built model has 
reproduced similar results $(165 \mathrm{~mm})$. Large deformation is observed around the penetrator due to a compaction of the concrete. The model also predicts a beginning of spalling forming on the back side of the target which cannot be observed on the impacted specimen. The concrete damage is characterised by diffuse failure on its first half and fewer large cracks on the second half. The diffuse damaged part can be easily ejected from the jacket sometime after the impact. The model projectile deceleration in Fig. 3 correlates with the test measurements obtained from the high-speed camera images. The use of $\gamma$-SPH discretisation is totally relevant for this application because it improves the modelling of projectile/target contact interaction. The concrete confinement is also better reproduced as no element is deleted.

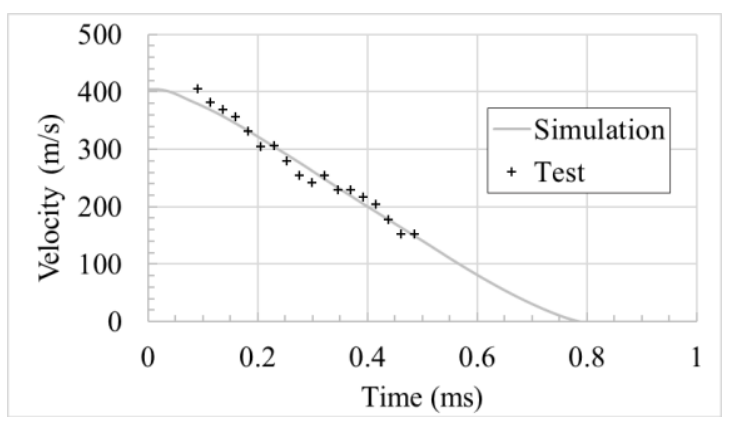

Fig. 3. Projectile deceleration during penetration

\section{Conclusion}

This paper shows some preliminary results of a down scaled KE penetrator impact on highly confined cylindrical concrete. Both characterisation tests and hypersonic impact tests are conducted to calibrate and evaluate a numerical model combining a $\gamma$-SPH discretisation with HJC material model. The first simulations give very good results in terms of penetrator/concrete interaction. Projectile depth of penetration and deceleration are reproduced by the model as well as the concrete damage. In perspective, the signals from the measurements (strain gages on the jacket, back face velocity measurement...) will be analysed to refine the model if needed. Additional perforation tests are planned with KEP with embedded accelerometers that should give complementary information on the penetration process. Simulations of all the remaining tested configurations will be conducted to evaluate the residual velocity in case of perforation and the robustness of the model for a range of impact velocity between 300 and $900 \mathrm{~m} / \mathrm{s}$.

\section{Acknowledgments}

The authors thank THIOT-INGENIERIE laboratory team for performing all the tests.

\section{References}

1. T.J. Holmquist, G.R. Johnson, W. Cook, “A Computational Constitutive Model for Concrete Subjected to Large Strains, High Strain Rates and High Pressures”, $14^{\text {th }}$ Int. Symp. on Ballistics, Québec, Canada, 1993.

2. A. Collé, J. Limido, J.P. Villa, “An Accurate SPH Scheme for Dynamic Fragmentation modelling”, $12^{\text {th }}$ International DYMAT Conference, EPJ Web of Conferences, 183, 01030, France, 2018. 